\title{
Hematologic Presentation of Metastatic Prostate Carcinoma: A Case Report on a Brca2 Mutated Young Patient
}

\author{
Samya Hamad Mehanna ${ }^{1, *}$, Fabio Kendi Kunitaki ${ }^{1}$ and Elisa Klug Hansen ${ }^{2}$ \\ ${ }^{1}$ Department of Pathlogy, Evangélico Mackenzie University Hospital, Curitiba, Paraná, Brazil \\ ${ }^{2}$ Mackenzie Evangélico College, Curitiba, Paraná, Brazil
}

\begin{abstract}
Prostatic adenocarcinoma is one of the most frequent malignancy amongst men, in most patients with advanced disease $(65-75 \%)$ end up developing bone metastases. One of the factors linked to such condition is the genectic variability of cancer types, the most aggressive of them are derived from a series of mutations in tumor suppressor genes. BRCA2 is a tumor suppressor gene, the presence of a mutation within this gene increases the chance of developing prostate cancer by more than 8 times, being present in approximately $5 \%$ of patients with advanced stages. Currently, many treatment strategies are effective for patients with prostate cancer who have germline/somatic modifications in the DNA restoration pathway mechanism for BRCA1 and BRCA2. Other than that, the diagnosis of a BRCA2 mutation may be helpful in terms of genetic counseling, risk assessement and early screening.
\end{abstract}

\section{Keywords}

Prostate cancer, Bone metastases, BRCA2

\section{Introduction}

Prostatic adenocarcinoma is one of the most frequent malignancy amongst men [1], and its risk increases significantly after the age of 50 [2] and approximately $50 \%$ of patients undergoing local radiotherapy or prostatectomy may have recurrence [1]. Current treatments have extended the overall survival by more than 10 years with a high cure rate [3], still, in most patients with advanced disease (65-75\%) end up developing bone metastases [1], mainly in the spine, pelvis, ribs and long bones [4].

The reason for metastasis development and non-survival in some individuals is related to treatment resistance [3]. One of the factors linked to such condition is the genetic variability of cancer types, the most aggressive of them are derived from a series of mutations in tumor suppressor genes. Furthermore, the bone microenvironment and its interactions may be responsible for numerous disseminations, hindering the treatment [3]. Wherefore, genomic analysis have been utilized not only for diagnostic purposes, but also used as a therapeutic optimization resource [5].

\section{Case Presentation}

A previously healthy 37-years-old man, reported to the Emergency Department with a chief complaint of weakness, hyporexia, a 32 kilos weight loss, associated with episodes of epistaxis, bruises and multifocal pain. On physical examination, he was pale and tachycardic, without lymph node enlargement, with asymmetrical breasts (right nodule) and splenomegaly. Laboratory data showed sever anemia with reticulocytosis, consistent with a leukoerythroblastic picture and a possible bone marrow invasion.

Imaging exams demonstrated alterations in the breast, prostate, seminal vesicles, testis, liver, lungs, spine and lymph nodes. The MRI of the pelvis stands out, with extensive prostate damage, with lymph node enlargement, in addition to multiple bone lesions suggestive of metastasis (Figure 1).

The bone marrow biopsy impression (Figure 2A) was of a poorly differentiated metastatic epithelial neoplasm.

The immunohistochemical study indicated primary prostate site by positivity for AE1/AE3, CK20, Racemase and PSA, with a high proliferative index (Ki67> 70\%), being negative for other markers related to pulmonary, gastrointestinal,

*Corresponding author: Samya Hamad Mehanna, Mackenzie Evangélico College, Rua Padre Anchieta, 2770, Bigorrilho, Curitiba, Paraná, Brazil, 80730-000

Accepted: December 23, 2021

Published online: December 27, 2021

Citation: Mehanna SH, Kunitaki FK, Hansen EK (2021) Hematologic Presentation of Metastatic Prostate Carcinoma: A Case Report on a Brca2 Mutated Young Patient. J Clin Surg Pathol 3(1):31-33 


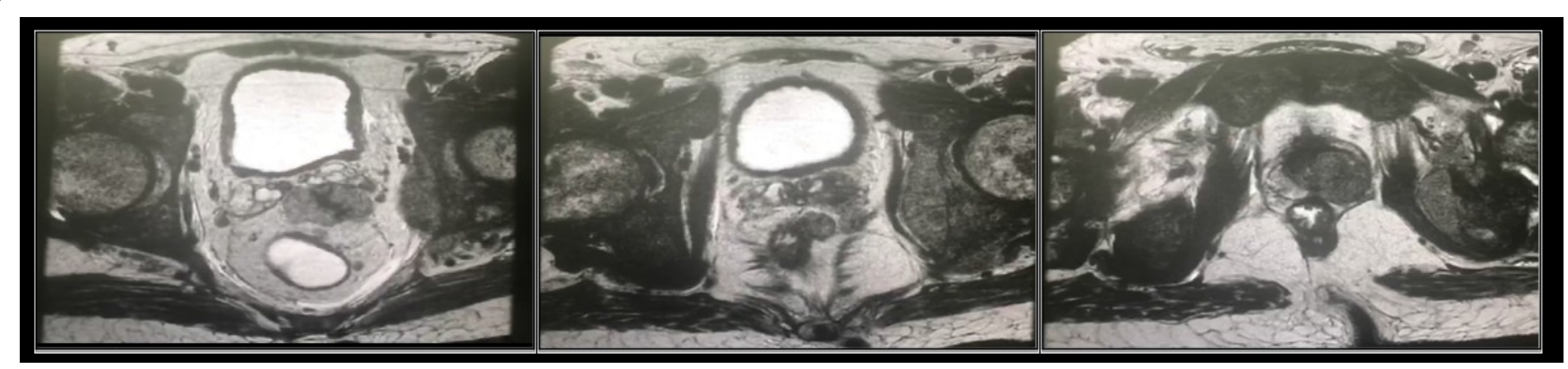

Figure 1: Pelvic MRI demonstrating a lesion centered on the left side of the prostate, showing signs of posterior extension to the ipsilateral seminal vesicle, with a malignant appearance. Lymph node enlargement and bone lesions suspicious for metastasis.

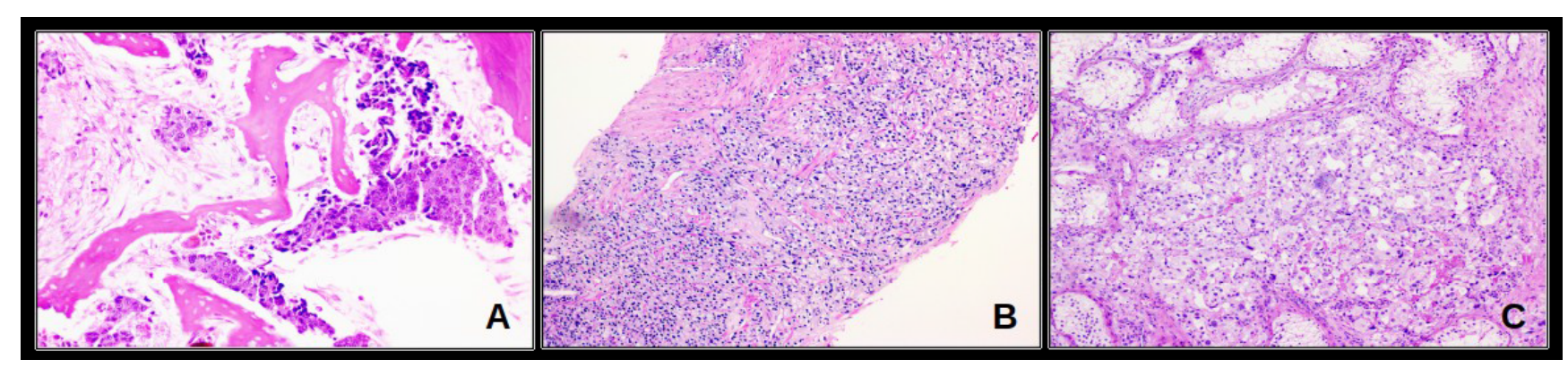

\section{Figure 2:}

2A: Bone marrow metastatic poorly differentiated carcinoma.

2B: Prostatic adenocarcinoma.

2C: Testicle with metastatic implant of prostatic origin.

renal, hepatic origin and germinal histogenesis. Subsequent prostate biopsy (Figure 2B) backed the diagnosis of an usual pattern acinar adenocarcinoma, Gleason $9(4+5)$, ISUP 5.

Hence, androgen blockade with Leuprolide and Bicalutamide, palliative radiotherapy for spinal pain, associated with chemotherapy with Docetaxel was instituted. Bilateral orchiectomy was also performed, and the left testicle presented prostate cancer metastasis (Figure 2C).

A pathogenic alteration with a BRCA2 gene mutation was confirmed on the genetic test. Afterwards, Carboplatin was associated to the treatment, later being replaced by Cisplatin, followed by clinical improvement.

\section{Discussion}

Less than $1 \%$ of the cases are detected in patients under the age of 50 years [6]. BRCA2 is a tumor suppressor gene, the presence of a mutation within this gene increases the chance of developing prostate cancer by more than 8 times, being present in approximately $5 \%$ of patients with advanced stages. Furthermore, mutations in BRCA2 havebeen reported to result in an aggressive pattern of disease, usually associated with Gleason greater than or equal to 8 , as well as the development of distant metastases, with a reduced survival rate, especially in metastatic cases resistant to castration [7]. Thus, genetic tests are essential for the assessment and screening of cancer risk, especially in younger patients, in addition to allowing genetic counseling for men in families with a hereditary condition of BRCA28.
Currently, many treatment strategies are effective for patients with prostate cancer who have germline/somatic modifications in the DNA restoration pathway mechanism for BRCA1 and BRCA2. Chemotherapy drugs are a beneficial treatment, and platinum-based cycles are highly sensitive for these patients [8]. Treatment with Docetaxel associated with Cisplatin is important due to the exposure that the mutation generates to the activity of these drugs, improving the prognosis [9]. Targeted therapies, such as PARP inhibitors (poly-ADP ribose polymerase), are used in BRCA-linked breast and ovarian tumors and are also recommended for BRCA patients with prostate cancer [8].

\section{Conclusion}

Prostate cancer has an insidious evolution, affecting mainly the elderly. Genetic research is advised on patients with aggressive disease and those with a strong family history of prostate cancer. The diagnosis of a BRCA2 mutation may be helpful in terms of genetic counseling, risk assessment and early screening. Lastly, molecular studies allow treatment optimization and prognostic improvement.

\section{References}

1. SAAD F (2005) Bone metastases in advanced prostate cancer. US Oncology Review 1: 1-5.

2. Wong SK, Nur-Vaizura Mohamad, Tijjani Rabiu Giaze, et al. (2019) Prostate cancer and bone metastases: The underlying mechanisms. Int J Mol Sci 20.

3. Logoghetis C, Michael J Morris, Robert Den, et al. (2018) Current perspectives on bone metastases in castrate-resistant prostate câncer. Cancer Metastasis Rev 37: 189-196. 
4. Shore N (2016) Bone metastases and mortality in protsate cancer, can we be doing more? Everyday Urology-Oncology Insights 1.

5. Sartor O, Bono JS (2018) Metastatic prostate cancer. N Engl J Med 378: 645-657.

6. World health organization (2016) Pathology and genetics of tumours of the urinary system and male genital organs. [s.I.] IARCPress.
7. Castro E, Chee Goh, David Olmos, et al. (2013) Germline BRCA mutations are associated with higher risk of nodal involvement, distant metastasis, and poor survival outcomes in prostate câncer. J Clin Oncol 31: 1748-1757.

8. Noor N Junejo, Sultan S AlKhateeb (2020) BRCA2 gene mutation and prostate cancer risk. Saudi Medical Journal 41: 9-17.

9. Alanee SR, Emily A Glogowski, Kasmintan A Schrader, et al. (2014) Clinical features and management of BRCA1 and BRCA2 associated prostate cancer. Front Biosci (Elite Ed) 6: 15-30. 